\title{
El autismo en los manuales diagnósticos internacionales: cambios y consecuencias en las últimas ediciones
}

\author{
Autism in international diagnostic manuals: Changes and consequences \\ in the last editions
}

\section{Palabras claves}

Autismo, TEA, CIE, DSM.

\section{Keywords}

Autism, TEA, CIE, DSM.

\author{
María Laura Grosso Funes \\ $<$ marialaura.grosso@unc.edu.ar> \\ Universidad Nacional de Córdoba. \\ Argentina
}

\section{Introducción}

El trastorno del espectro autista (TEA) ha pasado por diferentes descripciones en su clasificación nosográfica. Desde la creación del término autista hasta la actualidad, las descripciones del comportamiento de las personas con TEA han pasado por diferentes categorías de clasificación y por muchas discusiones en el ámbito de la salud mental hasta llegar a la actualidad, con una clasificación dimensional categorial del autismo como un trastorno del neurodesarrollo. La larga historia de modificaciones de denominación, clasificación y descripción se relaciona con cambios de paradigmas en cómo concebir y abordar la salud-enfermedad y con los avances tecnológicos que generan rupturas, continuidades e innovaciones en los modos de construir las teorías y posicionar los objetos a estudiar.

EI TEA mantiene los síntomas específicos clínicos identificados por Kanner (1943) y Asperger (1944; Wing, 1981). Los manuales internacionales que clasifican los trastornos mentales y otras patologías han modificado, en las últimas ediciones, la identificación, descripción de los síntomas, conductas y los criterios de diagnóstico del autismo que, actualmente, denominan TEA. A continuación, se presentan los manuales internacionales contextualizados (Lakatos, 1974) con sus continuidades y rupturas a modelos y paradigmas anteriores y las similitudes y diferencias entre ellos.

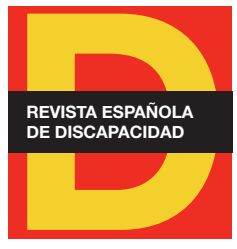

Para citar:

Grosso, M. L. (2021). El autismo en los manuales diagnósticos internacionales: cambios y consecuencias en las últimas ediciones. Revista Española de Discapacidad, 9(1), pp. 273-283.

Doi: <https://doi.org/10.5569/23405104.09.01.15> 
En este trabajo se abordarán los manuales que diagnostican el trastorno del espectro autista y se analizarán las posiciones desde donde se construye y sus repercusiones fuera del ámbito clínico. Los manuales son el punto de partida de los diagnósticos: documentos con valor legal. El alcance de esos documentos permite o impide el acceso a derechos como los educativos, terapéuticos y sociales.

\section{Los manuales}

\subsection{EI TEA en la Clasificación Internacional de Enfermedades y trastornos relacionados con la Salud Mental (CIE)}

Los manuales de la Organización Mundial de la Salud (OMS) y la CIE $(1990,2018)$ se utilizan en más de 110 países.

La CIE abarca una amplia variedad de condiciones de salud, cataloga las distintas patologías y trastornos con el fin de proporcionar un lenguaje común para informar y controlar las enfermedades, así como comparar y compartir datos siguiendo unos criterios estándares entre instituciones, regiones y países en distintos períodos de tiempo. Para ello, los términos diagnósticos se convierten en códigos alfanuméricos únicos para lesiones, enfermedades y causas de muerte.

Actualmente, la Organización Mundial de la Salud define la salud de una forma amplia dentro de un marco bio-psico-social, como un estado de completo bienestar físico, mental y social, no sólo como la ausencia de enfermedad. Para comprender y explicar la discapacidad hay dos modelos conceptuales diferentes. El modelo médico ve la discapacidad como un problema de la persona, causado por una enfermedad, un trauma u otras condiciones que inciden negativamente sobre la salud. El manejo y la "gestión" de la discapacidad se realizan a través de la cura de la enfermedad o la adaptación del individuo a su estado a través del cambio del comportamiento. En este caso, las decisiones políticas para afrontar la discapacidad elaboran respuestas principalmente médicas y sanitarias. Las decisiones sociales no apuntan a la integración del individuo con discapacidad. En el modelo social la discapacidad emerge como un problema de la sociedad. En este modelo la discapacidad no es un atributo sino un conjunto de condiciones causadas por el ambiente social. Por lo tanto, el manejo de la problemática requiere de la responsabilidad colectiva de toda la sociedad para aportar las modificaciones necesarias al ambiente y permitir la participación plena de las personas con discapacidad en todos los sectores de la vida social, por lo que la cuestión de la discapacidad se lleva adelante a nivel político con decisiones que repercuten a nivel social. La última CIE integra los modelos clínico y social y, por este motivo, se la define como una clasificación bio-psico-social, en donde los aspectos biológicos y psíquicos descienden del modelo médico y se unen al modelo social.

La CIE-11 (2018) es la última edición y reemplaza a la anterior clasificación, ClE-10 (1990), aunque entrará en vigor en el 2022. Se publica después de diez años de trabajo para actualizar las diferentes condiciones de salud y enfermedad, simplificar la estructura de codificación y facilitar el registro de las mismas. Por ejemplo, el TEA viene incluido en la categoría con código 6 de: trastornos mentales, comportamentales y del neurodesarrollo, específicamente en la subcategoría trastornos del neurodesarrollo: 6A02 Trastorno 
del espectro autista (Autism spectrum disorder). Esta clasificación es nueva y da cuenta de la inclusión de la perspectiva del neurodesarrollo en los trastornos. El trastorno es una entidad de diagnóstico que hace referencia a un conjunto de dificultades que presentan algunas personas, de carácter crónico y atribuibles a una atipicidad del funcionamiento intelectual general y de la conducta adaptativa en la línea de proceso evolutivo de la persona y en su integración bio-psico-social. Se los explica como dificultades desde los niveles biológico, cognitivo-emocional y conductual. Los TEA son así un grupo complejo de trastornos comportamentales y cognitivos del desarrollo cerebral, con una etiología que abarca factores ambientales y genéticos y, en la mayoría de los casos, hasta desconocidos.

La nueva clasificación adopta un enfoque de distinción dimensional-categorial. La perspectiva dimensional utiliza diversos valores ordenados en un continuo y evalúa varios niveles de algún atributo. Asimismo, aporta mayor información clínica (parámetros de intensidad, gravedad, duración, deterioro, uso de servicios). El enfoque del sistema categorial usa valores binarios ("sí-no"): presencia o ausencia. Tiene los problemas de añadir mayor complejidad al proceso clasificatorio y ser poco familiar al punto que, en la práctica clínica, se tienden a establecer distinciones categoriales para facilitar el etiquetado.

Las dimensiones que caracterizan al TEA son 2: dificultades en la interacción y comunicación social e intereses restringidos y conductas repetitivas.

La anterior clasificación, la CIE-10, tiene un enfoque basado en un modelo de clasificación psicopatológico; parte de la consideración de los estados de salud (enfermedad, lesiones, trastornos) según la etiología, con un paradigma predominantemente clínico.

El autismo pertenecía a la categoría de F84 Trastornos generalizados del desarrollo, TGD (PDD, Pervasive developmental disorders, en inglés) y agrupaba un conjunto de perfiles clínicos caracterizados por la tríada de anomalías cualitativas: 1. la interacción social recíproca, 2. patrones idiosincráticos de comunicación y el lenguaje y 3. un repertorio estereotipado, restringido y repetitivo de actividades e intereses. Estas anomalías cualitativas eran las características del funcionamiento de la persona afectada en todas estas situaciones.

En el CIE-11 se modifica la nomenclatura de trastornos generalizados del desarrollo (TGD) al TEA, conjuntamente con las subclasificaciones. El trastorno permanece y hace referencia a un comportamiento o grupo de síntomas identificables clínicamente, que producen malestar, disfunción o interferencia con las actividades que se espera que realice el individuo. La aceptación del término espectro enfatiza la diversidad y heterogeneidad de manifestaciones de un mismo atributo. Se incluye, en la clasificación actual del TEA, al síndrome de Asperger con otro nombre (ex F84.5) como 6A02.0, a otros trastornos generalizados del desarrollo dentro de la clasificación de TEA (ex F.84.8 y F.84.9) como 6A02.Y y 6A02.Z respectivamente. Se excluye del manual al autismo infantil (ex F84.0), ya que el autismo es una condición de por vida, al trastorno desintegrativo infantil (ex F84.3) porque tiene importantes problemas de validez y al síndrome de Rett (ex F84.2) porque pertenece a otro trastorno.

La ClE-11 modifica la nomenclatura categorial por una distinción dimensional que aporta pautas para distinguir entre autismo con y sin discapacidad intelectual y con ausencia o presencia grave/leve de lenguaje funcional. La CIE-11 también incluye la pérdida de competencias previamente adquiridas como una característica a tener en cuenta a la hora de efectuar un diagnóstico.

El lenguaje, que en la CIE-10 era una característica triádica, se ha eliminado como dimensión del TEA, pero ingresa como un especificador categorial según la presencia leve-grave o ausencia del mismo. También se 
señala la importancia de examinar sensibilidades sensoriales inusuales, algo común entre las personas con autismo. La CIE-11 pone menos énfasis en el tipo de juego que los niños realizan (variable cultural) y se concentra en si los menores siguen o se imponen reglas estrictas cuando juegan, un comportamiento que puede percibirse en cualquier cultura y que puede ser un signo de inflexibilidad, característica común en el TEA.

La CIE-11 recoge las características de género, tanto la relación o la proporción entre masculino y femenino como las variaciones que puede adoptar dependiendo del individuo. Los rasgos femeninos del TEA son diferentes a los masculinos, especialmente durante la infancia, y sus características singulares no están recogidas en ningún manual diagnóstico ni clasificatorio.

\subsection{EI TEA en el Diagnostic and Statistical Manual of Mental Disorders (DSM)}

En la América anglófona se usa exclusivamente el DSM creado por la American Psychiatric Association (APA) (desde 1953, con diferentes ediciones hasta llegar al 2013), como así también en algunos países latinoamericanos (Brasil: Lei Federal No 12.764, 2012; Venezuela; Colombia). En Argentina (Ley No 27.043) y en Uruguay (Ley $N^{\circ} 18.651$ ) se usan ambos.

EI DSM es un sistema de clasificación de la conducta anormal, se basa en criterios empíricos adoptados por la psicopatología, la psiquiatría y la psicología clínica, en general. Esta clasificación de los trastornos mentales posibilita el uso de un lenguaje común entre los investigadores y terapeutas para describir la psicopatología, facilitando la replicación de trabajos de investigación, las acciones interdisciplinares, la homologación de los tratamientos, los estudios epidemiológicos y el análisis de la efectividad de las terapias basadas en la evidencia.

La definición de trastorno mental para el DSM-5 es "un síndrome caracterizado por una perturbación clínicamente significativa en la cognición, la regulación emocional o la conducta del individuo que refleja una disfunción en los procesos psicológicos, biológicos o del desarrollo que subyacen al funcionamiento mental" (American Psychological Association, 2013). Predomina un punto de vista biológico en la perspectiva del manual. El paradigma presenta una visión centrada en el trastorno. El centrarse en la enfermedad supone partir de una situación de déficit puro sin considerar que la mayor parte de los trastornos mentales obedecen etiológicamente a mecanismos multifactoriales. Este modelo médico ve la discapacidad como un problema de la persona, causado directamente por una enfermedad, un trauma u otras condiciones de salud. Para este modelo, la persona necesita de un cuidado sanitario proporcionado por un tratamiento individual por parte de profesionales. El manejo y la "gestión" de la discapacidad se realiza a través de la cura de la enfermedad o la adaptación del individuo a su estado a través del cambio del comportamiento.

EI TEA está incluido en la clasificación del trastorno del neurodesarrollo, junto con las discapacidades intelectuales y los trastornos de la comunicación. Los TEA son así un grupo complejo del desarrollo cerebral, con causas multifactoriales.

Aunque el DSM-5 mantiene la estructura básica de clasificación categorial originada a partir del DSM-III, se reconoce que los trastornos mentales no siempre se ajustan a los límites de un único trastorno. Uno de los cambios del DSM-5 fue el reconocimiento oficial del carácter continuo de los trastornos mentales, es decir, la imposibilidad de establecer categorías continuas y discretas de los trastornos. Se integran en la estructura de clasificación categorial dos tipos de parámetros dimensionales: (a) los espectros o equivalen- 
tes dimensionales para diagnósticos (dimensiones psicopatológicas), y (b) las evaluaciones dimensionales (medidas de síntomas transversales, cross-cutting y medidas de gravedad).

Los criterios dimensionales del DSM-5 del TEA son dos:

a. Déficits persistentes en la comunicación y la interacción social, y

b. Patrones de comportamiento, intereses o actividades restringidas y repetitivas.

El DSM-5 no tiene una escala general de gravedad sino que su clasificación en leve, moderado o grave está dada por el nivel de soporte requerido. Para el diagnóstico se considera la gravedad en los deterioros de la comunicación social y en patrones de comportamientos restringidos. Los niveles de gravedad de los TEA del DSM-5 son: demasiado, mucho o algún apoyo.

También incluye un especificador cuando el TEA se asocia a una condición médica, genética o ambiental, lo cual requiere la inclusión de información sobre potenciales factores etiológicos (síndrome $\mathrm{X}$ frágil, exposición fetal al alcohol y epilepsia).

El DSM-5 facilita la observación de los criterios en la comunicación verbal y no verbal, pero es difícil evaluarlos en los niños pequeños, al igual que la relación con pares en niños menores de 2 años; lo mismo con las rutinas y los rituales fijos y restringidos, por lo cual se especifica en la evaluación aspectos del desarrollo como un patrón raro.

En el DSM-IV-TR el autismo estaba codificado en la clasificación 299 de los trastornos generalizados del desarrollo, sus equivalencias al $\mathrm{ClE}-10$. Estos trastornos son manifestaciones de entidades clínicamente separadas.

Uno de los cambios en el DSM- 5 respecto a la edición anterior es la unificación en TEA de todas las subclasificaciones de los trastornos generalizados del desarrollo: el autismo infantil ${ }^{1}$, trastorno global del desarrollo sin especificar y el Asperger. El síndrome de Rett y el trastorno desintegrativo de la infancia no aparecen en el DSM-5, ya que el primero tiene una base genética diferente del TEA y el segundo presenta problemas de validez científica.

Los criterios del DSM-IV-TR para diagnosticar el autismo eran 16. En la tabla 1 se comparan los criterios y las dimensiones de las últimas dos ediciones del DSM $(2002,2013)$.

1. El autismo no es una forma infantil de la esquizofrenia (como se pensó durante un largo tiempo) ni cambia estructuralmente en la edad adulta. Es, más bien, una condición long life. 
Tabla 1. Comparación de los criterios diagnósticos de los DSM

DSM-IV-TR (American Psychological Association, 2002)
$\begin{aligned} & \text { A. Un total de } 6 \text { (o más) ítems de (1), (2) y (3), con al menos } \\ & \text { dos de (1), y uno de (2) y de (3): }\end{aligned}$
dos de (1), y uno de (2) y de (3):

(1) Alteración cualitativa de la interacción social, en al menos por dos de las siguientes características:

(a) alteración de comportamientos no verbales: contacto ocular, expresión facial, posturas corporales y gestos reguladores de interacción social.

(b) incapacidad para desarrollar relaciones con compañeros al nivel de desarrollo.

(c) ausencia de tendencia espontánea para compartir con otros disfrutes, intereses y objetivos (no mostrar, traer o señalar objetos de interés).

(d) falta de reciprocidad social o emocional.

(2) Alteración cualitativa de la comunicación en al menos por dos de las siguientes características:

(a) retraso o ausencia del desarrollo de lenguaje oral (sin compensar con modos alternativos de comunicación, como gestos o mímica).

(b) aquellos con habla adecuada, alteración en la capacidad para iniciar o mantener una conversación.

(c) utilización estereotipada y repetitiva del lenguaje o lenguaje idiosincrásico.

(d) ausencia de juego realista espontáneo, variado, o juego imitativo social propio del nivel de desarrollo.

(3) Patrones de comportamiento, intereses y actividades restringidos, repetitivos y estereotipados, en al menos una de las siguientes características:

(a) preocupación por uno o más patrones estereotipados y restrictivos de interés que resulta anormal, sea en su intensidad, sea en su objetivo.

(b) inflexible a rutinas o rituales específicos, no funcionales.

(c) manierismos motores estereotipados y repetitivos (sacudir o girar las manos o dedos, o movimientos complejos de todo el cuerpo).

(d) preocupación persistente por partes de objetos.

B. Retraso o funcionamiento anormal en al menos una de las siguientes áreas, aparece antes de los 3 años de edad: (1) interacción social, (2) lenguaje usado en comunicación social o (3) juego simbólico o imaginativo.
DSM-5 (American Psychological Association, 2013)

A. Todos estos déficits en comunicación e interacción social en múltiples contextos, actuales o pasadas:

1. Déficit en reciprocidad social y emocional. Abarcan desde anormal acercamiento social e incapacidad de mantener alternancia en una conversación, reducción de intereses, emociones y afectos compartidos hasta ausencia total de iniciativa en la interacción social.

2. Déficit en conductas de comunicación no verbal que se usan en la comunicación social, abarcan desde una comunicación poco integrada, verbal como no verbal, anormalidades en el contacto visual y el lenguaje corporal o déficit en la comprensión y uso de la comunicación no verbal, hasta ausencia de expresiones o gestos faciales.

3. Déficit en el desarrollo y mantenimiento de relaciones adecuadas al grado de desarrollo, abarcan desde dificultades para mantener un comportamiento apropiado para diferentes contextos sociales, dificultades para compartir juegos imaginativos hasta la aparente ausencia de interés en otras personas.

Especificar la gravedad actual que se basa en deficiencias en la comunicación social y en patrones de conducta restrictivos o repetitivos.

B. Patrones de comportamiento, intereses o actividades restringidas y repetitivas, al menos dos de:

1. Habla, movimientos o manipulación de objetos estereotipada o repetitiva.

2. Excesiva fijación con las rutinas, los patrones ritualizados de conducta verbal y no verbal o excesiva resistencia al cambio.

3. Intereses altamente restrictivos y fijos de intensidad desmesurada.

4. Hiperreactividad o hiporreactividad a estímulos sensoriales o inusual interés en aspectos sensoriales (indiferencia al dolor/calor/frío, respuesta adversa a sonidos o texturas, sentido exacerbado del olfato o del tacto, fascinación por luces $u$ objetos que ruedan).

La gravedad basada en las deficiencias en la comunicación y patrones de conductas restrictivas o repetitivas. 


\begin{tabular}{|l|l|}
\hline DSM-IV-TR (American Psychological Association, 2002) & DSM-5 (American Psychological Association, 2013) \\
\hline $\begin{array}{l}\text { C. El trastorno no se explica mejor por la presencia de un } \\
\text { trastorno de Rett o de un trastorno desintegrativo infantil. }\end{array}$ & $\begin{array}{l}\text { C. Los síntomas deben estar presentes en el período } \\
\text { temprano del desarrollo (pueden no ser hasta que la } \\
\text { demanda social excede sus capacidades o compensadas por } \\
\text { estrategias aprendidas posteriormente). }\end{array}$ \\
\hline & $\begin{array}{l}\text { D. Síntomas causan limitaciones significativas a nivel social, } \\
\text { laboral u otras áreas de funcionamiento actual. }\end{array}$ \\
\hline $\begin{array}{l}\text { E. Las alteraciones no se explican mejor por discapacidad } \\
\text { intelectual o retraso global del desarrollo. Discapacidad } \\
\text { intelectual y TEA coexisten. Hacer diagnóstico comorbido } \\
\text { de TEA y discapacidad intelectual, comunicación social es } \\
\text { inferior al desarrollo general. }\end{array}$ \\
\hline $\begin{array}{l}\text { Nota: Las personas con diagnóstico de autismo del DSM-IV } \\
\text { deberían recibir diagnóstico de TEA. Aquellos con déficits } \\
\text { en la comunicación social, que no alcanzan los criterios de } \\
\text { TEA, deberían ser evaluados para considerar el trastorno de } \\
\text { comunicación social (pragmática). }\end{array}$ \\
\hline $\begin{array}{l}\text { Especificar si: } \\
\text { 1. Con o sin deficiencia intelectual. } \\
\text { 2. Con o sin deficiencia de lenguaje. } \\
\text { 3. Asociado a condición médica/genética conocida/factores } \\
\text { ambientales. } \\
\text { 4. Con catatonia. }\end{array}$ \\
\hline
\end{tabular}

Fuente: elaboración propia.

El afecto no se consideraba un descriptor en ninguna clasificación de los trastornos generalizados del desarrollo. Sin embargo, el DSM-5 introduce el rasgo que cualifica la relación social en tanto reciprocidad, no ya solo interacción. El afecto es uno de los objetos que mutuamente se intercambian en la correspondencia emocional. Los TEA Asperger interactúan socialmente, aunque manifiestan problemas en la dinámica misma de la relación, en entrar de un modo fluido en los intercambios interpersonales. Alternancia y compartir son rasgos que circunscriben al afecto $^{2}$ en una dinámica recíproca socioemocional.

A diferencia del DSM-IV-TR, cuyos criterios se centraban en la identificación de niños en edad escolar, el DSM-5 garantiza la identificación del trastorno en niños mayores. Según el DSM-5, los individuos con TEA presentan síntomas desde la infancia, aunque se los reconozcan después, lo que permite diagnosticar a las personas que solo manifiestan los síntomas cuando las exigencias sociales exceden su capacidad.

EI DSM-5 es más estricto que la edición anterior con respecto al diagnóstico, lo cual puede dar lugar a un aumento en la especificidad y disminución de falsos positivos a expensas de la disminución de la sensibilidad, especialmente para niños mayores, adolescentes y adultos, personas sin discapacidad intelectual, individuos con diagnóstico previo de Asperger o trastorno generalizado del desarrollo no especificado del DSM-IV-TR.

2. La larga historia del autismo como un trastorno de origen psicoafectivo (derivada de la lectura de Kanner sobre el contacto afectivo) ha creado discusiones y problemas en la historia de la psiquiatría y la psicología. La introducción del atributo aquí delimita el déficit no tanto en el objeto (afecto) sino en la dinámica de la relación (reciprocidad). 
El diagnóstico de TEA del DSM-5 se realiza en base a una dimensión de autismo, con diversos niveles de gravedad. El concepto de espectro es más claro que la orientación categorial.

Para la evaluación de los TEA, el DSM-5 tiene en cuenta el retraso cognitivo o alteraciones en el desarrollo del lenguaje, el retroceso en habilidades ya adquiridas y la falta de atención a un objeto y a una persona. La discapacidad intelectual y el TEA con frecuencia coinciden; para hacer diagnósticos de comorbilidades de un TEA y una discapacidad intelectual, la comunicación social ha de estar por debajo de lo previsto para el nivel general de desarrollo.

Las personas con autismo pueden tener comorbilidad con cualquier tipo de alteración del desarrollo, psicológica o psiquiátrica (TDAH, trastornos del movimiento u otras condiciones como la esquizofrenia). En el DSM-5 se pueden hacer los diagnósticos mencionados junto con el TEA.

EI DSM-5 posee criterios de clasificación dimensionales, organiza mejor las descripciones sintomáticas y las evaluaciones. Asimismo, la evaluación de las necesidades individuales de soporte es útil para los clínicos, para garantizar la atención educativa, social, de salud mental y de intervenciones médicas. Estos cambios suponen que el evaluador debería conocer los logros del desarrollo motor para cada edad y evaluar si las características de los síntomas, la precisión y la ejecución de los movimientos son óptimas o no, para determinar si interfieren en el desempeño global, ya que podrían afectar al cuidado personal como al juego de socialización y también al aprendizaje, porque frecuentemente se encuentra afectada la habilidad para escribir.

Todos estos cambios reducen la especificidad de algunas categorías diagnósticas. Los nuevos trastornos poseen un excesivo énfasis en las teorías médico-biológicas que apoyan o vienen promovidas por intereses de otros sectores, como son las ambiciones y lógicas de mercado (Parenti y Pambukdjian, 2016) del galenismo actual (Argañaraz, 2012, p. 152). En poblaciones vulnerables, niños y adolescentes se fomenta un exceso de prescripciones de fármacos (Frances, 2010). La expansión del sistema de nuevos trastornos y la reducción a dimensiones de algunas categorías podrían dar lugar a una inflación de diagnósticos, creando falsas epidemias de trastornos mentales (Frances, 2009).

\section{Conclusiones}

La CIE es una clasificación realizada por las Naciones Unidas, de libre acceso, al servicio de la salud pública. El DSM es un manual producido por una asociación profesional de una única disciplina de un solo país y en el que la asociación tiene finalidades económicas. La elaboración de la CIE se enmarca en un proceso global y multidisciplinar en diversas lenguas y tiene en cuenta la multiculturalidad mundial, mientras que el DSM se genera desde una perspectiva estadounidense y anglófona. La CIE tiene como objetivo ser de utilidad a los países miembros de la Organización Mundial de la Salud, así como a los servicios de atención sanitaria de primera línea, mientras que el DSM está mayormente dirigido al campo de la psiquiatría. Desde la Organización Mundial de la Salud se plantea que la definición de trastorno no puede ser llevada a cabo por una única organización profesional que representa a una única disciplina de la salud, en un contexto de un país concreto y que cuenta con intereses comerciales. 
La CIE-11 actualiza los criterios de diagnóstico del autismo, en la misma línea que el DSM-5. En este sentido, recoge el término único de Trastorno del espectro autista y engloba en esta única categoría a las anteriores: "Autismo", "Síndrome de Asperger", "Trastorno desintegrativo infantil” y "Otros trastornos generalizados del desarrollo no especificados" (Organizzazione Mondiale della Sanità , 2004). El cambio de nombre enfatiza la dimensionalidad del trastorno y la dificultad para establecer límites precisos entre los subgrupos. Ambos manuales consideran al TEA como un trastorno del neurodesarrollo, no ya un trastorno generalizado del desarrollo, ampliando la perspectiva del desarrollo como un complejo devenir atípico del fenotipo humano.

Con respecto a las características nucleares del TEA, la ClE-11 incluye las mismas categorías dimensionales que el DSM-5 (dificultades para la interacción y la comunicación social e intereses restringidos y comportamientos repetitivos), eliminando un tercer descriptor relacionado con el lenguaje. Ambas clasificaciones señalan también la necesidad de examinar sensibilidades sensoriales inusuales, característica descriptiva común entre las personas con autismo.

Hay diferencias entre los manuales CIE-11 y DSM-5. La clasificación de la OMS aporta pautas detalladas para distinguir entre autismo con y sin discapacidad intelectual; el DSM-5 solo señala que el autismo y la discapacidad intelectual podrían presentarse simultáneamente. La CIE-11 también incluye la pérdida de competencias previamente adquiridas como una característica a tener en cuenta a la hora de efectuar un diagnóstico. EI DSM-5 hace referencia explícita a la existencia de anomalías sensoriales, como la alta o baja sensibilidad a los sonidos o al tacto. La CIE no incluye la respuesta a los estímulos sensoriales como criterio, sino que lo considera un ítem más del patrón restrictivo y estereotipado de comportamientos, lo cual podría excluir a muchos individuos de ser considerados con este diagnóstico. La alternativa sería añadir una nota para aclarar que ciertas alteraciones sensoriales se encuentran presentes.

Por lo que respecta a la etapa de desarrollo infantil, la CIE-11 pone menos énfasis en el tipo de juego que los niños realizan centrándose en las reglas impuestas, un comportamiento que puede ser signo de inflexibilidad, característica del TEA.

Finalmente, ambos manuales hacen referencia al autismo aludiendo al término espectro. La inclusión responde al hecho de que, en la práctica cotidiana, se venía utilizando desde hace tiempo para designar la amplitud de manifestaciones en un continuo del trastorno, complejo y multiforme, que no presenta un patrón único sino que abarca un amplio rango de alteraciones de diferentes dimensiones y no como una categoría única. La contrapartida es que el concepto de espectro podría generar un efecto de epidemia (Frances, 2009) o hiperinclusividad del TEA al tener menos descriptores categoriales y basarse en solo dos dimensiones. 


\section{Referencias bibliográficas}

American Psychological Association (1994). Diagnostic and statistical manual of mental disorders (4th). American Psychological Association.

American Psychological Association (2002). Manual diagnóstico y estadístico de los trastornos mentales. Texto revisado (DSM-IV-TR). Masson.

American Psychological Association (2013). Diagnostic and statistical manual of mental disorders (5th ed.). American Psychological Association.

Argañaraz, J. (2012). Ruptura y continuidad de Lacan con Freud (desde Lakatos). Universidad de Córdoba, Facultad de Filosofía y Humanidades.

Argentina. Ley № 27.043, de 15 de diciembre de 2014, que declara de interés nacional el abordaje integral e interdisciplinario de las personas que presentan trastornos del espectro autista (TEA).

http://servicios.infoleg.gob.ar/infoleglnternet/anexos/240000-244999/240452/norma.htm.

Asperger, H. (1944). Die ‘Autistischen Psychopathen' im Kindesalter. Archiv für Psychiatrie und Nervenkrankheiten, (117), pp. 76-136.

Brasil. Lei № 12.764, de 27 de dezembro de 2012, Institui a Política Nacional de Proteção dos Direitos da Pessoa com Transtorno do Espectro Autista; e altera o $\S 3^{\circ}$ do art. 98 da Lei $n^{\circ} 8.112$, de 11 de dezembro de 1990. Diário Oficial da União, 28 de dezembro de 2012, p. 2. https://www2.camara.leg.br/legin/fed/lei/2012/ lei-12764-27-dezembro-2012-774838-publicacaooriginal-138466-pl.html.

Frances, A. (2009). Whither DSM-V? Br. J. Psychiatry, 195(5), pp. 391-399. http://doi.org/10.1192/bjp.bp.109.073932.

Frances, A. (2010). Alert to the research community-be prepared to weigh in on DSM-V. Psychiatric times, 27, pp. $1-2$.

Kanner, L. (1943). Autistic disturbances of affective contact. Nervous Child, (2), pp. 217-250.

Lakatos, I. (1974). Popper y los problemas de demarcación e inducción. En Escritos filosóficos I. Alianza.

Organización Mundial de la Salud (1990). CIE-10. Décima edición de la Clasificación Internacional Estadística de Enfermedades y Problemas Relacionados con la Salud. Editorial Médica Panamericana. http://ais.paho.org/ classifications/Chapters/.

Organización Mundial de la Salud (2018). Clasificación Internacional de Enfermedades, $11^{a}$ revisión. Organización Mundial de la Salud. https://icd.who.int/es.

Organizzazione Mondiale della Sanità (2001). Classificazione Internazionale del Funzionamento, della Disabilità e della Salute. Erickson.

Parenti, M. y Pambukdjian, M. F. (2016). Análisis de la Ley del Trastorno del Espectro Autista (TEA) y su adecuación a los paradigmas de infancia y Salud Mental. VIII Congreso Internacional de Investigación y Práctica Profesional en Psicología. XXIII Jornadas de Investigación. XII Encuentro de Investigadores en Psicología del MERCOSUR. Facultad de Psicología, Universidad de Buenos Aires, Argentina. https://www.aacademica. org/000-044/571.pdf. 
Uruguay. Ley $N^{\circ} 18.651$, de 19 de febrero de 2010, de protección integral de personas con discapacidad. Diario Oficial, 9 de marzo de 2010, núm. 27932. https://www.impo.com.uy/bases/leyes/18651-2010/49.

Wing, L. (1981). Asperger's syndrome: A clinical account. Psychological Medicine, (11), pp. 115- 130. 